\title{
The Shocking Variability Of Exoplanet Transits
}

\author{
J. Llama, A. A. Vidotto, M. Jardine, K. Wood and R. Fares \\ SUPA. University of St Andrews. North Haugh. St Andrews. KY16 9SS. UK \\ email: joe.llama@st-andrews.ac.uk
}

\begin{abstract}
Asymmetries in exoplanet transits are proving to be a useful tool for furthering our understanding of magnetic activity on both stars and planets outside our Solar System. Near-UV observations of the WASP- 12 system have revealed asymmetries in the timing of the transit when compared with the optical light curve. In this paper we review a number of reported asymmetries and present work simulating near-UV transits for the hot-Jupiter hosting star HD 189733.
\end{abstract}

Keywords. stars: activity, coronae, individual (WASP-12, HD 189733), magnetic fields, planetary systems, winds

\section{Introduction}

Since the discovery of the first planet orbiting a star other than the Sun nearly twenty years ago the field of exoplanetary science has rapidly grown with both ground and space based surveys having found approximately 900 exoplanets ( $h t t p: / / w w w . e x o p l a n e t . e u)$. So called hot-Jupiters which are found to be orbiting within fractions of an au of their host star continue to challenge our ideas of planet formation and evolution. With the improvement of the telescopes used to find exoplanets we are now able to begin characterising the atmospheres of the planets. To this end, asymmetries in the transit light curves of exoplanets are proving to be incredibly useful tools for learning about both exoplanetary atmospheres but also the host star itself (Vidotto et al. 2011).

There have been many reports in the literature of "bumps" being recorded in the transit light curve of many exoplanets (see for example, Sanchis-Ojeda et al. (2011)). These bumps are believed to have been caused by the planet occulting a star spot on the surface of the host star. Because star spots are darker than their surroundings, the fractional loss of light as the planet transits over them will become less and so a positive bump will be recorded in the light curve. Missions such as Kepler provide continuous observations of many stars which therefore allow us to track the evolution of star spots on the surface of other stars that host misaligned planets. This will enable us to build up a picture of stellar butterfly diagrams (Llama et al. 2012).

Another asymmetry that has been detected is the presence of an early-ingress in the near-UV light curve taken with HST of the hot-Jupiter WASP-12b when compared to the optical data. The near-UV light curve shows the transit beginning before the optical transit, but finishing at the same time (Fossati et al. 2010). A number of potential solutions have been proposed to explain the presence of such an asymmetry. One solution suggests that because WASP-12b is a very large planet orbiting very close to its host star, it may have overflowed it's Roche lobe (Lai et al. 2010). This would result in an accretion stream from the planet onto the star which may manifest as an asymmetry in the light curve. Another solution is the presence of a magnetosphere around the planet (Vidotto et al. 2010). The interaction between the Solar wind and each of the Solar system planets 

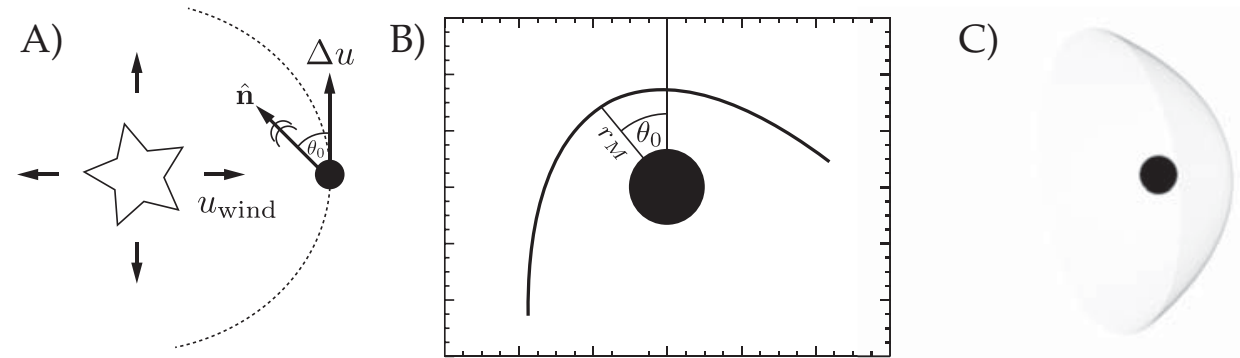

Figure 1. Image A) shows the geometry of the shock (adapted from Vidotto et al. (2010)). The distance $r_{M}$ is the distance to the nose of the shock. The angle $\theta_{0}$ is the angle made between the azimuthal direction of the planet and the vector $\hat{\mathbf{n}}$, the direction of the shock nose. The vector $-\hat{\mathbf{n}}$ is the direction the stellar wind impacts on the planet. B) shows a typical shock shape in two-dimensions using the model of Wilkin (1996). C) shows the shock geometry in three-dimensions.

results in the formation of a shock surrounding each of the planets. For planets that are located far from their host star, the shock will form directly between the planet and the star, a so-called "dayside shock". For close-in planets, such as hot Juipiters, however, an "ahead shock" will form which will occult the stellar disc before the planet which may cause an early-ingress in the transit light curve (Vidotto et al. 2010). If the density of shocked material is high enough then this may show as the presence of additional absorption in the light curve. Llama et al. (2011) modelled the near-UV light curve of WASP-12b from Fossati et al. (2010) and were able to fit the observations with a simple shock model.

In this paper we model a planet and bow shock transiting over a simulated star. We use magnetic maps of the bright K-dwarf HD 189733 (which due to its proximity and relative brightness has been extensively studied) to simulate the stellar wind conditions around the planet transiting planet HD 189733b. From these simulations we are able to prescribe the geometry and density of the shock that should form and simulate near-UV light curves.

\section{The Model}

We couple numerical simulations of stellar winds with magnetic imaging of HD 189733 to predict the stellar wind conditions around the planet HD $189733 \mathrm{~b}$ in order to investigate how the geometry of the resultant shock may vary as the planet orbits around the star. We use magnetic surface maps of HD 189733 from June 2007 and also a year later in July 2008 (Fares et al. 2010). They use the tomographic method known as ZeemanDoppler Imaging (ZDI) to reconstruct the large-scale magnetic field on the surface of the star.

The magnetic surface maps are used as one of the boundary conditions in our stellar wind simulation. We use BATS-R-US, a three-dimensional mangetohydrodynamic (MHD) numerical code developed at The University of Michigan (Powell et al. 1999). BATS-R-US solves the ideal MHD equations for the mass density $\rho$, the gas pressure $p$, the velocity of the plasma $\mathbf{u}=\left(u_{r}, u_{\theta}, u_{\phi}\right)$ and the magnetic field intensity $\mathbf{B}=\left(B_{r}, B_{\theta}, B_{\phi}\right)$. The output from the wind simulation is a full three-dimensional grid that allows us to determine the local conditions experienced by the transiting planet HD 189733b.

The formation of a bow shock is a direct consequence of the relative velocities between the interaction of the planetary magnetic field and the stellar wind occurring at supersonic speeds. The distance and geometry of the shock is related to the local stellar wind 

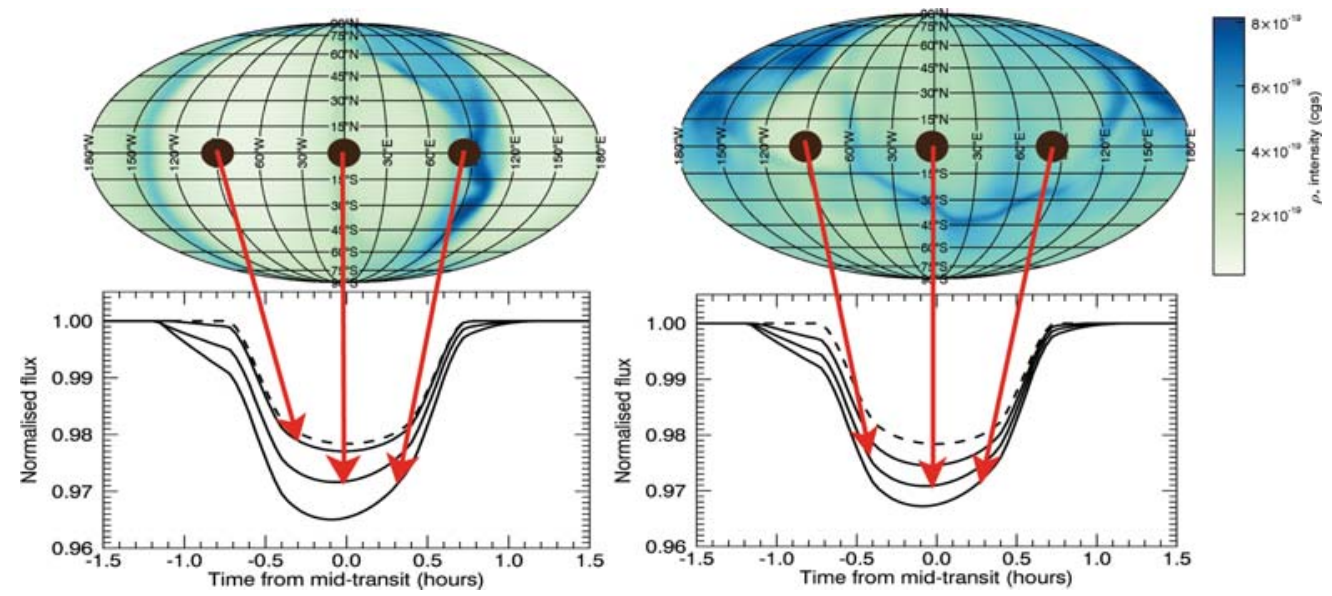

Figure 2. Results of our simulations: June 2007 (left panel) and July 2008 (right panel). Top panel shows the stellar wind density at the orbital distance of the planet. The transit depth and timing is clearly dependent on the local stellar wind conditions for near-UV transits. We find the transits to be highly variable, with certain wind conditions causing the transit to be almost indistinguishable from the optical transit, whilst in very dense regions of the wind the transit depth is much deeper and also begins much sooner due to the presence of denser, shocked material.

properties near the planet. The shape of the shock is described in two-dimensional polar co-ordinates $(r, \theta)$ with the planet centred at $(0,0)$ as:

$$
R\left(r_{M}, \theta\right)=\frac{r_{M}}{\sin \theta} \sqrt{3\left(1-\frac{\theta}{\tan \theta}\right)}
$$

where $r_{M}$ is the distance to the nose of the shock (Wilkin 1996). The angle the shock makes with the azimuthal direction of the planetary motion is defined as $\theta_{0}$, and is determined by the geometry of the stellar wind impacting on the planet:

$$
\theta_{0}=\arctan \left(\frac{u_{r}}{\left|u_{\text {planet }}-u_{\varphi}\right|}\right)
$$

where $u_{\text {planet }}$ is the orbital motion of the planet, and $u_{r}$ and $u_{\varphi}$ are the radial and azimuthal stellar wind speeds respectively (Vidotto et al. 2010). Figure 1 shows a cartoon depiction of the shock model.

\section{Results}

Figure 2 shows our results. The top image shows the stellar wind density at the orbit of HD 189733b for June 2007 (left) and July 2008 (right). The bottom panel shows three resultant near-UV transits (solid lines) and the optical model for comparison (dashed). The results clearly show that the depth and timing of the transit is dependent on the density of the stellar wind and therefore multiple observations may reveal different transit shapes and help us understand the variability of the stellar wind.

\section{References}

Fares, R., Donati, J., \& Moutou, C., et al. 2010, MNRAS 406, 409

Fossati, L., Haswell, C., Froning, C., et al. 2010, ApJ 714, L222 
Lai D., Helling C., \& van den Heuvel E. P. J. 2010, ApJ, 721, 923

Llama, J., Jardine, M., Mackay, D. H., \& Fares, R. 2012, MNRAS 422, L72

Llama J., Wood K., Jardine M., Vidotto A. A., et al. , 2011, MNRAS 416, L41

Powell K. G., Roe P. L., Linde T. J., et al. 1999, Journal of Computational Physics, 154, 284

Sanchis-Ojeda R., Winn J. N., Holman M. J., et al. 2011, ApJ, 733, 127

Vidotto A. A., Jardine M., \& Helling C. 2010, ApJ, 722, L168

Vidotto, A. A, Jardine, M., \& Helling, Ch. 2011, MNRAS, 414, 1573

Vidotto, A. A, Llama, J., Jardine, M., et al. 2011, Astronomische Nachrichten, 332, 1055

Wilkin F. P. 1996, ApJ, 459, L31

\section{Discussion}

JACKSON: What sort of interactions and/or degeneracies would there be between a bow shock and planetary evaporation?

Llama: There is an inherent degeneracy between a bow shock and planetary evaporation, both of which would provide an asymmetry in the light curve. However, the presence of planetary evaporation does not rule out the possibility of a bow shock also being present.

Tinney: Your models look at the absorption produced by a shock. Have you thought about whether the emission signature of these shocks would be detectable?

LLAMA: In principle you could certainly look for emission; however, this emission would have to be above any stellar emission and so this may cause issues.

BARstow: How realistic is the assumption that the magnetic field of HD $189733 \mathrm{~b}$ is similar to Jupiter's?

LlAma: We know very little about the magnetic fields of exoplanets and so it seems reasonable to assume the magnetic field to be as simple as possible, i.e. a dipole with the same intensity as Jupiter's. The model can simply be scaled to account for the specific field strength should a better estimate be found.

RAFIKOV: How do you treat mass loading of field lines in your magnetic model? Does the planet always move through super-Alfvénic parts of the wind?

LLAMA: From the stellar wind model we predict a mass loss rate of $\dot{M}_{\star}=4 \times 10^{-13} M_{\odot} / \mathrm{yr}$. At the orbital radius of HD 189773b our model predicts the wind is indeed super-Alfvénic.

PUDRITZ: M-stars are highly variable and have strong magnetic field and X-ray activity. Would it be possible to probe the magnetic field in Super-Earths around M-stars?

Llama: One certainly could look for asymmetries in Super-Earths around M-stars. The activity of the star may cause some issues. We discuss this in Vidotto et al. (2011).

ShKOLNIK: Could the WASP-12b early ingress be due to the planet grazing over a bright hot spot on the star?

LlAmA: A bright spot certainly would cause an asymmetry in the light curve. However, the bright spot would not cause an early-ingress, rather it would simply cause additional absorption whilst the planet occulted the spot. 